\title{
Atividade biológica em solo salino sódico saturado por água sob cultivo de Atriplex nummularia ${ }^{1}$
}

\author{
Biological activity in saline sodic soil saturated by water under cultivation of Atriplex \\ nummularia
}

\author{
Karen Cristina Fialho dos Santos ${ }^{2 *}$, Marina Steffane Lopes da Silva ${ }^{3}$, Luís Eduardo da Silva ${ }^{3}$, Marcelo Alves \\ Miranda $^{4}$ e Maria Betânia Galvão dos Santos Freire ${ }^{5}$
}

\begin{abstract}
Resumo - A salinidade constitui-se um sério fator limitante à agricultura, pois a maioria das culturas agrícolas é sensível à salinidade, sendo muito afetadas pelos efeitos nocivos dos sais. As espécies halófitas do gênero Atriplex caracterizam-se pela elevada tolerância a seca e salinidade do solo, sendo uma alternativa viável para reduzir impactos negativos da salinidade, associada à biomassa microbiana. Portanto, o objetivou-se com este trabalho analisar a atividade microbiana em um solo salino sódico do Agreste de Pernambuco, na presença e ausência de plantas de Atriplex nummularia. Para isso, realizaramse amostragens de solo a $20 \mathrm{~cm}$ de profundidade em diferentes situações: 1 e 2$)$ nas proximidades das raízes $(20 \mathrm{~cm}$ do colo da planta) nos tratamentos com poda e sem poda; 3 e 4) no ponto intermediário do espaçamento entre plantas (125 cm) nos tratamentos com poda e sem poda; 5) e na área sem cultivo das plantas (tratamento testemunha); em período chuvoso. Nessas amostras foram feitas análises de $\mathrm{C}$ e $\mathrm{N}$ da biomassa microbiana, respiração basal, e C e $\mathrm{N}$ total dos solos. Observou-se que o tratamento testemunha teve resultados diferenciados em relação aos outros tratamentos, indicando que sem as plantas de atriplex a microbiota do solo é bastante afetada pela alta concentração de sais. Verificou-se, ainda, uma tendência de aumento da atividade microbiana com a proximidade das raízes de plantas podadas de Atriplex, mostrando que, além de ser uma planta viável para a fitorremediação de solos salinizados, ainda promove uma melhoria da microbiota do solo.
\end{abstract}

Palavras-chave - Biomassa microbiana. Salinidade dos solos. Plantas halófitas.

\begin{abstract}
The salinity constitutes a serious limiting factor for agriculture, because most crops are sensitive to salinity, being much affected by the harmful effects of salts. Halophyte species of the genus Atriplex are characterized by high tolerance to drought and soil salinity, a viable alternative to reduce negative impacts of salinity, associated with the microbial biomass. Therefore, the aim of this study was to assess the microbial activity in saline sodic soil of the Agreste of Pernambuco, in the presence and absence of plants of Atriplex nummularia. For that, there were samples of soil to $20 \mathrm{~cm}$ depth in different situations: 1 and 2$)$ in the proximity of roots $(20 \mathrm{~cm}$ from the stem of each plant) in treatments with and without pruning, 3 and 4$)$ at the midpoint of plant spacing $(125 \mathrm{~cm}$ ) in treatments with and without pruning, 5) bare soil (control treatment), in the rainy season. These samples were analyzed for $\mathrm{C}$ and $\mathrm{N}$ microbial biomass, basal respiration and $\mathrm{C}$ and $\mathrm{N}$ content of soils. It was observed that the control treatment had different results compared with other treatments, indicating that without plants of atriplex the soil microbiota is highly affected by the high salt concentrations. There was also a tendency for increased microbial activity with proximity of pruned plant roots of atriplex, showing that, besides being a viable plant for phytoremediation of salinized soils, still promotes an improvement of soil microbes.
\end{abstract}

Key words - Microbial biomass. Soil salinity. Halophytes.

\footnotetext{
* Autor para correspondência

Recebido para publicação em 31/08/2010; aprovado em 31/01/2011

Trabalho submetido e selecionado no primeiro Simpósio Brasileiro de Salinidade realizado de 12-15/10/2010 em Fortaleza; Parte da Dissertação de Mestrado do primeiro autor apresentada ao Programa de Pós-Graduação em Ciência do Solo/UFRPE

2Embrapa Mandioca e Fruticultura, Rua Embrapa, s/n, Chapadinha, Cruz das Almas-BA, Brasil, 44.380-000, karen@enpmf.embrapa.br ${ }^{3}$ Graduando de Agronomia/UFRPE, Bolsista PIBIC/FACEPE, Recife-PE, Brasil, marina.steffane@hotmail.com, luiseduardo.les87@yahoo.com.br ${ }^{4}$ Universidade Federal Rural de Pernambuco, Recife-PE, Brasil, marcelo_ufrpe@yahoo.com.br

${ }^{5}$ Departamento de Agronomia, DEPA/UFRPE, Rua Dom Manoel de Medeiros, s/n, Dois Irmãos, Recife-PE, Brasil, 52.171-900, betania@depa.ufrpe.br
} 


\section{Introdução}

A salinidade, tanto dos solos como das águas, é uma das principais causas da queda de rendimento das culturas (FLOWERS, 2004) devido aos efeitos de natureza osmótica, tóxica ou nutricional (VIANA et al., 2004). Em escala mundial, existe uma área de aproximadamente 400 milhões de hectares de terras utilizadas na agricultura cuja produção vem sendo severamente restringida pela salinidade (BOT et al., 2000).

As halófitas são plantas adaptadas a altos níveis de salinidade e têm capacidade de acumular quantidades elevadas de sais em seus tecidos e de extraí-los dos solos, sendo muito utilizadas na recuperação de solos afetados por sais, técnica denominada de "fitorremediação". Para o sucesso da fitorremediação em solos salino-sódicos, as plantas devem apresentar tolerância ao excesso de sais e alta produção de biomassa nesta condição. Além disso, devem acumular elevados teores de sais na parte aérea, visando a possibilitar a remoção dos sais com a colheita das plantas (ZHU, 2001). A fitoextração de sais utilizando plantas halófitas é uma alternativa de baixo custo para recuperação de solos salinos, não agressiva ao ambiente e que não requer alto nível tecnológico para sua efetivação (LEAL et al., 2008).

Uma das plantas mais utilizadas em fitorremediação de solos salinos e sódicos é a da espécie Atriplex nummularia, planta halófita, reconhecidamente tolerante ao estresse salino e hídrico, bem como produtora de grande biomassa vegetal, o que a torna capaz de extrair quantidades consideráveis de sais desses solos (LEAL et al., 2008; PORTO et al., 2006; SOUZA, 2010). Ao se estabelecer no solo, esta planta interage com ele, modificando suas propriedades químicas, físicas e biológicas e recebendo a influência das mesmas sobre o crescimento e a capacidade de extração de sais, sendo de elevada importância o conhecimento dessas interações no processo de recuperação.

A biomassa microbiana é a principal fonte de enzimas, sendo responsável pela quase totalidade da atividade biológica no solo, catalisando as transformações bioquímicas, representando fonte e dreno de carbono e troca de nutrientes entre a atmosfera e o ecossistema solo-planta (MOREIRA; SIQUEIRA, 2002). Mudanças significativas na quantidade de biomassa podem ser detectadas muito antes que alterações na matéria orgânica total possam ser percebidas, possibilitando a adoção de medidas de correção antes que a perda da qualidade do solo seja mais severa (TÓTOLA; CHAER, 2002).

A diversidade microbiana tem sido relacionada com a qualidade do solo. No entanto, estudos recentes apontam para a necessidade de investigação das interações e atividades metabólicas dos microrganismos nos processos ecológicos do solo (TAYLOR et al., 2002). Isso poderia ser o diferencial no sucesso da fitorremediação de solos afetados por sais, comparandose com o solo sem o cultivo da atriplex. Portanto, o objetivo desse trabalho foi realizar a avaliação da atividade microbiana em solo salino-sódico, saturado por água, do Agreste de Pernambuco cultivado com Atriplex nummularia em condições de campo.

\section{Material e métodos}

Para a análise da atividade microbiana, amostras de solo foram coletadas em um experimento de campo, com o cultivo de plantas de Atriplex nummularia instalado há onze meses, visando à recuperação de Neossolo Flúvico salino-sódico, localizado no Município de Pesqueira, Pernambuco ( $8^{\circ} 34^{\prime} 17^{\prime \prime}$ de Latitude Sul, $37^{\circ} 1$ '20" de Longitude Oeste) (SOUZA, 2010). As principais caracterizações químicas do solo no início do experimento (setembro/2008) eram: $\mathrm{pH}=9,1$, CEes $=21,49 \mathrm{dS} \mathrm{m}{ }^{-1}, \mathrm{Na}^{+}$solúvel $=277,29 \mathrm{mmol}_{\mathrm{c}} \mathrm{L}^{-1}$, $\mathrm{Cl}^{-}$solúvel $=223,78$ mmol $_{\mathrm{c}} \mathrm{L}^{-1}$ e PST $=30,51 \%$, todas as análises químicas seguiram a metodologia proposta pela EMBRAPA (PAULA; DUARTE, 1997). O A coleta foi realizada no período chuvoso (agosto/2009), quando o solo da área encontrava-se saturado por água. $\mathrm{O}$ experimento foi conduzido em delineamento experimental em blocos casualizados, com três tratamentos: planta cultivada sem regime de poda, planta sob regime de poda aos seis e doze meses e o tratamento testemunha, sem cultivo de Atriplex, em quatro repetições. Foram coletadas amostras de solo na camada de 0-20 cm em locais distintos na área útil das parcelas de cada bloco: 1 e 2) nas proximidades das raízes (20 cm do colo da planta) nos tratamentos com poda e sem poda, respectivamente; 3 e 4) no ponto intermediário do espaçamento entre plantas $(125 \mathrm{~cm})$ nos tratamentos com poda e sem poda, respectivamente; 5) e na área sem cultivo das plantas (tratamento testemunha). Como o experimento foi montado em blocos casualizados, no total, foi feita coleta de solo em 5 pontos, sendo 4 blocos distintos, resultando em 20 amostras.

Imediatamente após a coleta, ainda no campo, as amostras foram armazenadas sob refrigeração e transportadas ao laboratório para as análises. No laboratório, foram submetidas à secagem parcial, tendo em vista a saturação do solo por água, com posterior tratamento por peneiramento para desagregação dos torrões. Antes das análises referentes à atividade biológica, a umidade nas amostras foi ajustada para $60 \%$ da capacidade de campo com a finalidade de ativar o metabolismo da biomassa presente no solo. 
A respiração basal microbiana (RBS) foi determinada pelo método da estimativa do $\mathrm{CO}_{2}$, onde este é adsorvido em $\mathrm{NaOH}$ presente em potes fechados e posteriormente determinado por titulação com HCl (ALEF; NANNIPIERI, 1995). O carbono e o nitrogênio da biomassa microbiana (CBM e NBM) foram determinados pelo método da fumigação extração, onde foi realizada a análise da biomassa microbiana extraível em solução aquosa de sulfato de potássio $\left(\mathrm{K}_{2} \mathrm{SO}_{4}\right)$ a $0,5 \mathrm{~mol} \mathrm{~L}^{-1}$ (VANCE et al., 1987). $\mathrm{O}$ carbono total do solo (COT) foi mensurado pelo método adaptado por Yeomans e Bremner (1988) e o nitrogênio total do solo (NT) foi determinado pelo método adaptado de Bremner e Mulvaney (1982) e Tedesco et al. (1995).

Com os resultados obtidos foram calculados o quociente metabólico $\left(\mathrm{qCO}_{2}\right)$, representado pela razão (respiração basal)/(carbono da biomassa microbiana), relação entre o $\mathrm{CO}_{2}$ acumulado e o total do CBM a qual prediz que na medida em que a biomassa microbiana se torna mais eficiente em utilizar os recursos do meio, menos $\mathrm{C}$ é perdido como $\mathrm{CO}_{2}$ pela respiração, podendo este ser incorporado aos tecidos microbianos; o quociente microbiano (qMIC), calculado pela relação entre o carbono da biomassa microbiana e o carbono orgânico total do solo, segundo a expressão qMIC $=\mathrm{CBM} / \mathrm{COT}$, que indica quanto do carbono está imobilizado na forma microbiana; a relação NBM/ NT que expressa quanto do nitrogênio está contido nos microrganismos; a relação entre o carbono e o nitrogênio da biomassa (CBM/NBM); e a relação entre o carbono e o nitrogênio total do solo $(\mathrm{C} / \mathrm{N})$.

Os resultados do trabalho foram analisados através da análise multivariada e das medidas de estatística descritiva, considerando os parâmetros média (tendência central) e coeficiente de variação (variabilidade). Os locais de amostragem de solo foram confrontados pela análise de componentes principais e pela análise de agrupamento. Antes da realização da técnica de agrupamento, todos os dados foram devidamente normalizados. A seleção dos CPs (Componentes Principais) se deu através da análise da variância de acordo com os autovalores gerados pela matriz padronizada, de tal forma que o maior autovalor foi associado ao primeiro componente principal (CP), o segundo maior autovalor ao segundo $\mathrm{CP}$, e assim por diante, até que o menor autovalor foi associado ao último $\mathrm{CP}$, colocando os primeiros como os mais importantes. Sendo assim, os primeiros componentes principais gerados pela ACP explicaram a maior parte da variância dos dados originais. Conforme critério de seleção dos componentes citados preferiu-se, neste trabalho, adotar a retenção dos componentes que explicaram mais de $70 \%$ da variância total (SANTOS, 2010).
Para a geração dos dendrogramas resultantes das análises de agrupamento, utilizaram-se a distância euclidiana média como coeficiente de similaridade e o algoritmo de WARD como método de agrupamento. Para realizar o corte do dendrograma utilizou-se uma das etapas da análise de agrupamento que, através de um gráfico gerado com as distâncias de ligação entre os grupos formados, definiu com maior precisão o ponto de corte no dendrograma em questão. Dessa forma, o corte efetuado no dendrograma, que determinou o número de grupos de acordo com uma maior similaridade, foi realizado de acordo com a maior distância (maior salto) com que os grupos foram analisados (FERREIRA, 2008). Todos os procedimentos estatísticos multivariados foram realizados com o software proposto por Hill e Lewicki (2007) - STATISTICA 8.0.

\section{Resultados e discussão}

A matriz de correlação obtida através da Análise de Componentes Principais (ACP) das variáveis biológicas do solo mostra um número significativo de correlações com valores altos entre algumas variáveis estudadas (TAB. 1).

Correlações altas entre estas variáveis podem ser importantes e explicar relações fundamentais para a manutenção da estabilidade da microbiota do solo. Pelos valores das correlações encontradas foi possível verificar que as variáveis RBS, COT, NT, qMIC, qCO , dependendo da variável com a qual estão relacionadas, puderam justificar melhor o comportamento da biota no solo por conta de seus altos valores de correlação que refletem a interrelação entre a atividade e a população microbiana do solo.

Como exemplo, pode-se analisar as relações positivas, do $\mathrm{C}$ e do $\mathrm{N}$ totais do solo em relação à $\mathrm{RBS}$ que indica quanto do aumento da respiração basal está relacionada ou é explicada pelos aumentos nos teores de $\mathrm{C}$ e $\mathrm{N}$ do solo.

A RBS responde sensivelmente às mudanças de fatores ambientais e, por isso, é uma quantificação importante, tendo em vista que representa a atividade biológica geral do solo. Nas correlações positivas entre a RBS e COT e o NT, há uma indicação de maiores concentrações desses elementos no solo, que proporcionam maior concentração de microrganismos e, consequentemente, maior taxa de respiração no solo. A importância dessa relação é alta, pois solos com maior biomassa de microrganismos conseguem promover melhores condições para o estabelecimento de plantas (TU et al., 2006). 
Tabela 1 - Matriz de correlação das variáveis biológicas obtidas em Neossolo Flúvico coletado no período chuvoso (agosto/2009), no município de Pesqueira, PE

\begin{tabular}{|c|c|c|c|c|c|c|c|c|c|c|}
\hline & RBS & $\overline{\mathrm{CBM}}$ & $\mathrm{COT}$ & qMIC & $\mathrm{qCO} 2$ & NBM & $\mathrm{CBM} / \mathrm{NBM}$ & NT & $\mathrm{C} / \mathrm{N}$ & $\mathrm{NBM} / \mathrm{N}$ \\
\hline $\mathrm{RBS}^{1}$ & 1,00 & $-0,47$ & 0,81 & $-0,80$ & 0,89 & 0,34 & $-0,68$ & 0,79 & 0,18 & 0,08 \\
\hline $\mathrm{CBM}^{2}$ & & 1,00 & $-0,14$ & 0,88 & $-0,76$ & 0,52 & $-0,30$ & $-0,26$ & 0,39 & 0,79 \\
\hline $\mathrm{COT}^{3}$ & & & 1,00 & $-0,54$ & 0,50 & 0,73 & $-0,84$ & 0,98 & $-0,03$ & 0,42 \\
\hline $\mathrm{qMIC}^{4}$ & & & & 1,00 & $-0,91$ & 0,09 & 0,15 & $-0,60$ & 0,15 & 0,42 \\
\hline $\mathrm{qCO}_{2}{ }^{5}$ & & & & & 1,00 & $-0,10$ & $-0,30$ & 0,52 & 0,11 & $-0,33$ \\
\hline $\mathrm{NBM}^{6}$ & & & & & & 1,00 & $-0,84$ & 0,62 & 0,31 & 0,91 \\
\hline $\mathrm{CBM} / \mathrm{NBM}^{7}$ & & & & & & & 1,00 & $-0,74$ & $-0,36$ & $-0,73$ \\
\hline $\mathrm{NT}^{8}$ & & & & & & & & 1,00 & $-0,21$ & 0,27 \\
\hline $\mathrm{C} / \mathrm{N}^{9}$ & & & & & & & & & 1,00 & 0,54 \\
\hline $\mathrm{NBM} / \mathrm{N}^{10}$ & & & & & & & & & & 1,00 \\
\hline
\end{tabular}

${ }^{1}$ Respiração basal (mg C-CO $\mathrm{kg}^{-1}$ solo hora- $\left.{ }^{-1}\right) ;{ }^{2}$ Carbono da biomassa microbiana (mg de C-CBM kg-1 solo seco); ${ }^{3}$ Carbono orgânico total do solo (dag kg-1); ${ }^{4}$ Quociente microbiano (\%); ${ }^{5}$ Quociente metabólico (mg C-CO $\mathrm{mg}^{-1} \mathrm{C}-\mathrm{CBM}$ dia $\left.{ }^{-1}\right) ;{ }^{6}$ Nitrogênio da biomassa microbiana (mg de NNBM kg-1 solo seco); ${ }^{7}$ Relação entre o carbono e o nitrogênio da biomassa microbiana; ${ }^{8}$ Nitrogênio total do solo (dag $\left.\mathrm{kg}^{-1}\right) ;{ }^{9} \mathrm{Relação} \mathrm{entre} \mathrm{o}^{-1}$ carbono e o nitrogênio totais do solo; ${ }^{10}$ Relação entre nitrogênio da biomassa microbiana e nitrogênio total do solo

Pode-se, ainda, observar a alta correlação positiva entre o COT e o NT, aproximando-se do valor máximo da correlação. O carbono total tem grande influência sobre as propriedades químicas, disponibilidade de nutrientes e atividade microbiana no solo (BAYER; MIELNICZUK, 2008). Um aumento da atividade microbiana provavelmente promoverá crescimento na população de microrganismos capazes de fixar nitrogênio, de forma que uma associação entre estes elementos é de fundamental importância para a manutenção da microbiota e da fertilidade do solo.

Altas correlações negativas, que indicam aumento de uma variável em função da diminuição da outra, foram observadas entre a RBS e o qMIC, entre o qMIC e o $\mathrm{qCO}_{2}$, e entre o $\mathrm{qCO}_{2}$ e o CBM. Todas as relações envolvem o CBM, indicando que esta é uma variável fundamental e decisiva para a manutenção da atividade microbiana.

Como o $\mathrm{qCO}_{2}$ é a relação entre a RBS e o CBM, um alto valor de RBS relacionado a um baixo valor de CBM resulta em um alto valor de $\mathrm{qCO}_{2}$, gerando possivelmente resultados de correlação positivos e altos entre $\mathrm{qCO}_{2}$ e RBS, como o demonstrado na matriz de correlação (TAB. 1). Este resultado é diferente do encontrado por Bezerra et al. (2008) que encontraram uma correlação negativa e baixa $\left(-0,2537^{\text {ns }}\right)$ para as mesmas variáveis citadas $\left(\mathrm{qCO}_{2}\right.$ e RBS). Por analogia, o valor negativo da correlação entre o $\mathrm{CBM}$ e o $\mathrm{qCO}_{2}$ mostra o inverso entre essas duas variáveis, enquanto o $\mathrm{CBM}$ e o qMIC se relacionam positivamente.
Solos com elevados teores de sais, como o deste estudo, podemapresentarlimitações ao desenvolvimento de plantas e microrganismos que podem promover a queda da população microbiana e, portanto, de sua atividade. Por outro lado, como os teores de COT e NT estão correlacionados positivamente com a RBS do solo, isso levaria à diminuição nessas variáveis acompanhando a RBS do solo (TAB. 2).

Baixas relações $\mathrm{C} / \mathrm{N}$ indicam maior taxa de decomposição dos resíduos orgânicos do que em relações mais elevadas (AITA; GIACOMINI, 2003). Esta relação variou entre 7,73 e 14,96 entre os solos estudados, dependendo da localização em relação à planta de Atriplex nummularia, com valores médios entre 10,33 e 11,51 (TAB. 2).

Observou-se maior relação $\mathrm{C} / \mathrm{N}$ no solo sem interferência da planta (Testemunha) em relação aos solos localizados próximos às raízes, indicando maior decomposição da matéria orgânica nas proximidades das raízes da Atriplex, provavelmente, em função da maior incidência de microrganismos promotores da decomposição. Em contrapartida, o valor máximo da relação $\mathrm{C} / \mathrm{N}$ encontrado na $\mathrm{CPR}$, que deveria estar menor por conta da alta população microbiana, ficou próximo do valor verificado no tratamento testemunha.

É possível que isto tenha ocorrido em função da recuperação da planta à poda drástica a qual foi submetida, poda esta que deve ter imobilizado os microrganismos em prol da possível reestabilização da biomassa, fazendo com que estes apenas mantivessem a atividade de decomposição, após um equilíbrio local. 
Tabela 2 - Medidas estatísticas descritivas dos atributos biológicos das amostras de solos coletadas em período chuvoso, correspondentes aos pontos SRSP (solo coletado a $125 \mathrm{~cm}$ da planta sem poda), SRCP (solo coletado a $125 \mathrm{~cm}$ da planta com poda), CPR (solo coletado a $20 \mathrm{~cm}$ do colo da planta com poda), SPR (solo coletado a $20 \mathrm{~cm}$ do colo da planta sem poda), T (testemunha)

\begin{tabular}{|c|c|c|c|c|c|}
\hline Variável biológica & Amostra & Média & Mínimo & Máximo & $\mathrm{CV}(\%)$ \\
\hline \multirow{5}{*}{$\begin{array}{c}\mathrm{RBS}^{1} \mathrm{mg} \mathrm{C}-\mathrm{CO}_{2} \mathrm{~kg}^{-1} \\
\text { solo hora }\end{array}$} & SRSP & 0,364 & 0,103 & 0,736 & 85,850 \\
\hline & SRCP & 0,531 & 0,207 & 0,753 & 45,120 \\
\hline & SPR & 1,407 & 0,631 & 2,637 & 61,120 \\
\hline & CPR & 1,351 & 1,128 & 1,897 & 27,248 \\
\hline & $\mathrm{T}$ & 0,497 & 0,166 & 0,856 & 56,983 \\
\hline \multirow{5}{*}{$\begin{array}{l}\mathrm{CBM}^{2} \text { mg C-CBM } \\
\mathrm{kg}^{-1} \text { solo seco }\end{array}$} & SRSP & 89,312 & 27,259 & 184,303 & 81,778 \\
\hline & SRCP & 63,101 & 26,050 & 120,417 & 67,355 \\
\hline & SPR & 77,712 & 37,173 & 130,502 & 57,443 \\
\hline & CPR & 62,651 & 36,990 & 94,856 & 42,563 \\
\hline & $\mathrm{T}$ & 124,489 & 103,158 & 135,184 & 11,832 \\
\hline \multirow{5}{*}{$\mathrm{COT}^{3}$ dag kg ${ }^{-1}$} & SRSP & 0,891 & 0,647 & 1,186 & 31,896 \\
\hline & SRCP & 0,872 & 0,634 & 1,014 & 20,807 \\
\hline & SPR & 1,320 & 0,698 & 1,760 & 35,620 \\
\hline & CPR & 1,011 & 0,691 & 1,696 & 45,691 \\
\hline & $\mathrm{T}$ & 0,936 & 0,642 & 1,426 & 38,125 \\
\hline \multirow{5}{*}{$\mathrm{qMIC}^{4} \%$} & SRSP & 0,013 & 0,002 & 0,028 & 97,119 \\
\hline & SRCP & 0,008 & 0,003 & 0,015 & 73,236 \\
\hline & SPR & 0,006 & 0,002 & 0,011 & 54,747 \\
\hline & CPR & 0,006 & 0,005 & 0,009 & 27,863 \\
\hline & $\mathrm{T}$ & 0,015 & 0,009 & 0,021 & 40,653 \\
\hline \multirow{5}{*}{$\begin{array}{l}\mathrm{qCO}_{2}{ }^{5} \mathrm{mg} \mathrm{C}-\mathrm{CO}_{2} \\
\mathrm{mg}^{-1} \mathrm{C}-\mathrm{CBM} \mathrm{dia}^{-1}\end{array}$} & SRSP & 0,006 & 0,001 & 0,014 & 99,223 \\
\hline & SRCP & 0,013 & 0,002 & 0,025 & 75,377 \\
\hline & SPR & 0,019 & 0,012 & 0,026 & 31,527 \\
\hline & CPR & 0,026 & 0,013 & 0,051 & 66,739 \\
\hline & $\mathrm{T}$ & 0,004 & 0,001 & 0,006 & 54,446 \\
\hline \multirow{5}{*}{$\begin{array}{c}\mathrm{NBM}^{6} \text { mg N-NBM } \\
\mathrm{kg}^{-1} \text { de solo seco }\end{array}$} & SRSP & 7,411 & 3,565 & 12,742 & 58,851 \\
\hline & SRCP & 6,100 & 1,804 & 14,710 & 97,569 \\
\hline & SPR & 25,074 & 18,833 & 31,953 & 23,054 \\
\hline & CPR & 7,232 & 3,692 & 9,224 & 34,373 \\
\hline & $\mathrm{T}$ & 20,731 & 8,793 & 50,339 & 95,517 \\
\hline \multirow{5}{*}{$\mathrm{CBM} / \mathrm{NBM}^{7}$} & SRSP & 17,706 & 4,010 & 51,695 & 128,403 \\
\hline & SRCP & 23,155 & 2,467 & 66,734 & 126,935 \\
\hline & SPR & 3,148 & 1,391 & 4,767 & 54,433 \\
\hline & CPR & 11,084 & 4,010 & 25,691 & 89,416 \\
\hline & $\mathrm{T}$ & 9,330 & 2,685 & 15,164 & 56,473 \\
\hline \multirow{5}{*}{$\mathrm{NT}^{8}$ dag kg ${ }^{-1}$} & SRSP & 0,087 & 0,055 & 0,116 & 28,800 \\
\hline & SRCP & 0,081 & 0,070 & 0,093 & 11,934 \\
\hline & SPR & 0,124 & 0,074 & 0,168 & 30,818 \\
\hline & CPR & 0,094 & 0,060 & 0,171 & 55,040 \\
\hline & $\mathrm{T}$ & 0,081 & 0,065 & 0,116 & 29,865 \\
\hline
\end{tabular}


Continuação Tabela 2

\begin{tabular}{cccccc}
\hline & SRSP & 10,333 & 7,734 & 11,688 & 18,024 \\
& SRCP & 10,794 & 7,582 & 12,816 & 21,113 \\
$\mathrm{C} / \mathrm{N}^{9}$ & $\mathrm{SPR}$ & 10,747 & 7,318 & 13,551 & 27,118 \\
& $\mathrm{CPR}$ & 11,209 & 9,250 & 14,086 & 19,199 \\
& $\mathrm{~T}$ & 11,506 & 8,961 & 14,960 & 23,358 \\
\hline \multirow{3}{*}{$\mathrm{NBM} / \mathrm{N}^{10} \%$} & $\mathrm{SRSP}$ & 0,010 & 0,004 & 0,023 & 88,396 \\
& $\mathrm{SRCP}$ & 0,008 & 0,003 & 0,019 & 99,828 \\
& $\mathrm{SPR}$ & 0,023 & 0,015 & 0,043 & 58,715 \\
& $\mathrm{CPR}$ & 0,010 & 0,002 & 0,014 & 55,626 \\
& $\mathrm{~T}$ & 0,029 & 0,010 & 0,077 & 109,802 \\
\hline
\end{tabular}

${ }^{1}$ Respiração basal; ${ }^{2}$ Carbono da biomassa microbiana; ${ }^{3}$ Carbono orgânico total do solo; ${ }^{4}$ Quociente microbiano; ${ }^{5}$ Quociente metabólico; ${ }^{6} \mathrm{Nitrogenio}$ da biomassa microbiana; ${ }^{7}$ Relação entre o carbono e o nitrogênio da biomassa microbiana; ${ }^{8}$ Nitrogênio total do solo; ${ }^{9}$ Relação entre o carbono e o nitrogênio totais do solo; ${ }^{10}$ Relação entre nitrogênio da biomassa microbiana e nitrogênio total do solo

Os resultados da relação CBM/NBM foram, em sua maioria, maiores do que aqueles observados por Barreto et al. (2008) que, trabalhando com solos cultivados com eucalipto, chegaram a valores médios que variaram entre 2,13 e 3,91 .

Estes valores médios indicam a presença de elevadas populações microbianas no solo estudado neste trabalho, sugerindo que os microrganismos presentes, mesmo em ambiente extremamente salino $\left(\right.$ CEes $=23,65 \mathrm{dS} \mathrm{m}^{-1}$ ), estão adaptados, possivelmente por possuírem mecanismo(s) de halotolerância aos sais presentes no solo.

Na Tabela 3 estão apresentados os autovalores, as percentagens das variâncias associadas aos CPs e a percentagem cumulativa. Neste caso, observa-se que a CP1, juntamente com a CP2, explicaram $86,36 \%$ da variação dos dados obtidos. Por isso, um estudo de todas as variáveis com apenas a informação dessas duas CPs não ocasionaria perdas relevantes de informações.

A importância de uma variável é analisada pela sua correlação com os CPs selecionados. Essa avaliação é feita através dos pesos das variáveis calculados pela $\mathrm{ACP}$, de forma que quanto maior o valor modular do peso da variável analisada maior a correlação com o CP a ela associada. Deste modo, é possível identificar quais variáveis estão correlacionadas com cada $\mathrm{CP}$ e qual a importância de cada variável para os objetivos da pesquisa (SANTOS, 2010).

Utilizando a mesma consideração feita para a Tabela 3, na Tabela 4 são apresentadas as correlações que foram mais importantes para o estudo das variáveis, ou seja, aquelas que se correlacionaram com os CPs 1 ou 2. Houve uma distribuição entre as variáveis biológicas estudadas quanto à associação ao CP1 ou CP2 (TAB. 4).

De posse dos valores das variáveis geradas para os CPs estudadas anteriormente, foram gerados valores dos locais de amostragem, também conhecidos por Scores que demarcam os pontos relacionados a cada local de amostragem em um gráfico de pontos. Como visto nas Tabelas 3 e 4, os dois CPs utilizados geraram um gráfico bidimensional para as amostras de solo coletadas no período chuvoso (FIG. 1).

Tabela 3 - Autovalores e variâncias dos componentes principais obtidas com os atributos biológicos do solo coletado no período chuvoso

\begin{tabular}{ccccc}
\hline Componente principal & Autovalor & \% da variância total & Autovalor cumulativo & \% Cumulativa \\
\hline CP1 & 4,86 & 48,57 & 4,866 & 48,57 \\
CP2 & 3,78 & 37,79 & 8,64 & 86,36 \\
CP3 & 1,17 & 11,75 & 9,81 & 98,11 \\
CP4 & 0,19 & 1,89 & 10,00 & 100,00 \\
\hline
\end{tabular}


Tabela 4 - Pesos dos atributos biológicos analisados para cada componente principal (CP) obtido para solos coletados em período chuvoso

\begin{tabular}{crr}
\hline Variáveis & \multicolumn{1}{c}{$\mathrm{CP} 1$} & \multicolumn{1}{c}{$\mathrm{CP} 2$} \\
\hline $\mathrm{RBS}^{1}$ & $\mathbf{- 0 , 9 4 2 8 8 1}$ & $-0,222211$ \\
$\mathrm{CBM}^{2}$ & 0,294644 & $\mathbf{0 , 9 4 7 6 8 7}$ \\
$\mathrm{COT}^{3}$ & $\mathbf{- 0 , 9 5 6 8 7 5}$ & 0,149786 \\
qMIC $^{4}$ & 0,688478 & $\mathbf{0 , 6 9 1 7 0 2}$ \\
$\mathrm{qCO}_{2}^{5}$ & $\mathbf{- 0 , 7 1 2 4 7 9}$ & $-0,592189$ \\
$\mathrm{NBM}^{6}$ & $-0,597368$ & $\mathbf{0 , 7 6 2 4 7 1}$ \\
$\mathrm{CBM}^{5} / \mathrm{NBM}^{7}$ & $\mathbf{0 , 8 1 2 3 7 2}$ & $-0,535570$ \\
$\mathrm{NT}^{8}$ & $\mathbf{- 0 , 9 2 7 2 5 6}$ & 0,010399 \\
$\mathrm{C} / \mathrm{N}^{9}$ & $-0,116138$ & 0,468377 \\
$\mathrm{NBM} / \mathrm{N}^{10}$ & $-0,305938$ & $\mathbf{0 , 9 4 4 5 4 2}$ \\
\hline
\end{tabular}

${ }^{1}$ Respiração basal (mg C-CO $\mathrm{kg}^{-1}$ solo hora-1); ${ }^{2}$ Carbono da biomassa microbiana (mg de C-CBM kg-1 solo seco); ${ }^{3}$ Carbono orgânico total do solo (dag kg-1 ${ }^{-1}$; ${ }^{4}$ Quociente microbiano (\%); ${ }^{5}$ Quociente metabólico (mg $\mathrm{C}-\mathrm{CO}_{2} \mathrm{mg}^{-1} \mathrm{C}^{-\mathrm{CBM}}$ dia $^{-1}$ ); ${ }^{\circ}$ Nitrogênio da biomassa microbiana (mg de N-NBM kg-1 solo seco); ${ }^{7}$ Relação entre o carbono e o nitrogênio da biomassa microbiana; ${ }^{8}$ Nitrogênio total do solo $\left(\mathrm{dag} \mathrm{kg}^{-1}\right) ;{ }^{9}$ Relação entre o carbono e o nitrogênio totais do solo; ${ }^{10}$ Relação entre nitrogênio da biomassa microbiana e nitrogênio total do solo

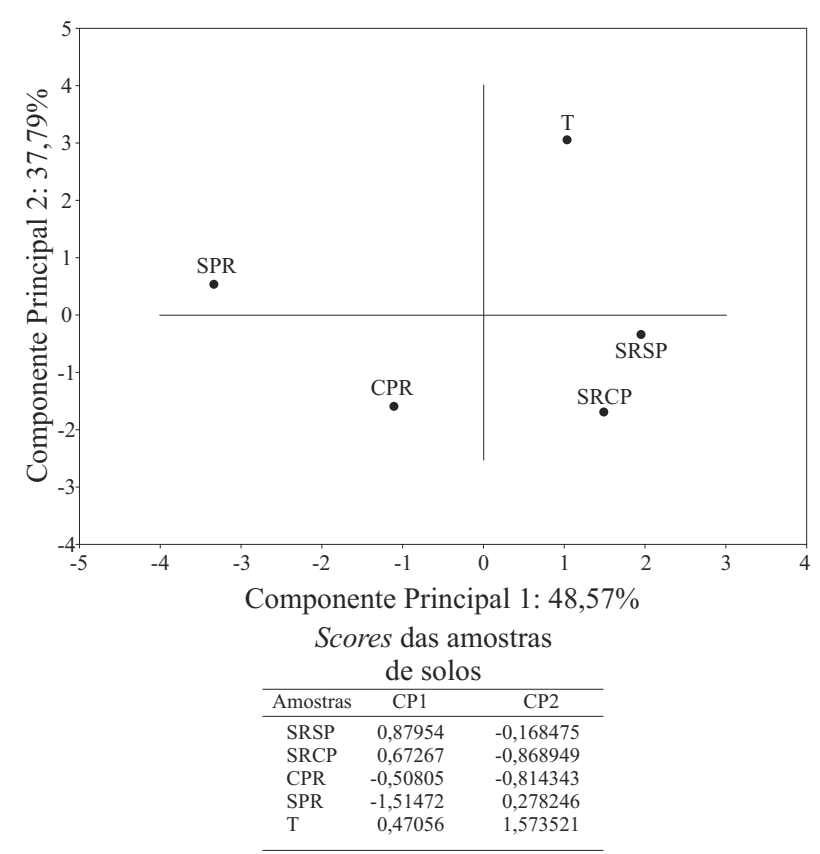

Figura 1 - Representação gráfica dos scores das posições de amostragem SRCP (125 cm da planta com poda), SRSP (125 cm da planta sem poda), CPR (20 cm da planta com poda), SPR (20 cm da planta sem poda) e T (testemunha), obtidos para as amostras de Neossolo Flúvico salino-sódico de Pesqueira, PE. Proximidade entre tratamentos indica semelhança de acordo com a análise de componentes principais
Observa-se, na Tabela 4, que as variáveis RBS, $\mathrm{COT}, \mathrm{qCO}_{2}$ e NT apresentam correlação negativa quanto ao $\mathrm{CP} 1$, mostrando que estas têm os seus valores médios aumentados quando indo da direita para esquerda do gráfico (FIG. 1). Já a variável CBM/ NBM correlacionou-se positivamente, tendo seu valor médio aumentado da esquerda para a direita do gráfico. Quanto ao CP2, as variáveis CBM, qMIC, NBM e $\mathrm{NBM} / \mathrm{N}$ correlacionaram-se positivamente, indicando que seus valores médios aumentam de baixo para cima do gráfico.

Sabe-se que valores positivos para o CP1 tendem a posicionar um ponto do lado direito do gráfico e os negativos, do lado esquerdo, enquanto, para o CP2, valores positivos posicionam o ponto mais acima do gráfico e os negativos, mais abaixo da linha central deste (FIG. 1).

Correlações positivas com o CP1 e negativas no CP2 aproximaram os usos SRSP e SRCP (FIG. 1). As correlações negativas do CP1 foram as mais importantes pelo distanciamento das posições SPR e CPR da T. Sendo que este último, provavelmente, ficou isolado por conta dos valores positivos em ambos os CPs para o CBM e qMIC, que pode ter ocorrido em função do alto grau de estresse pelo qual os microrganismos vem passando nos solos sob condição extremamente salina. Isso fica mais nítido ao se observar o dendrograma que representa os resultados obtidos por meio da análise de agrupamento (AA), em que as amostras de SRCP e SRSP se aproximam, distanciando-se da testemunha e, mais ainda, das amostras de SPR e CPR (FIG. 2).

A amostra de solo coletada no tratamento testemunha, pelo fato de não ter a influência das raízes, e ser um solo extremamente salino, permaneceu isolado, com redução da microbiota do solo naquelas condições, tendo em vista que o solo sob a ação da fitoextração por Atriplex nummularia apresenta melhores condições de adaptação e sobrevivência aos microrganismos em solos salinos. Em trabalho realizado com Atriplex nummularia e irrigação destas com rejeitos salinos, Pereira et al. (2004) observaram que houve um aumento da atividade microbiana, estimulada pelo plantio da Atriplex em campo.

As amostras coletadas a $20 \mathrm{~cm}$ das plantas isolaram-se em grupos distintos em função do corte realizado durante a poda, indicando que este trato cultural nas plantas interfere na atividade biológica no solo próximo às raízes, possivelmente, em consequência de alterações na tentativa de restabelecimento das condições homeostáticas locais por parte da planta, alterando o desenvolvimento da microbiota do solo. 


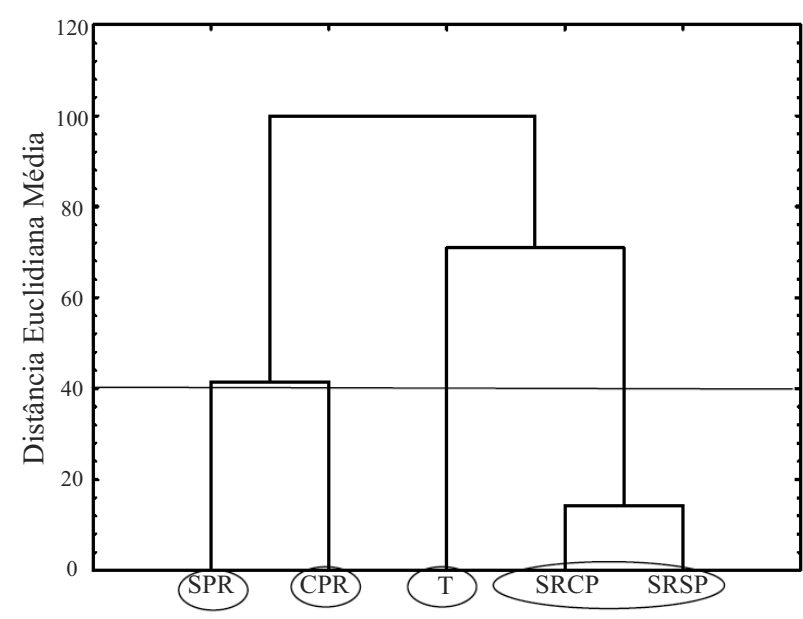

Figura 2 - Dendrograma resultante da análise de grupamento das posições de amostragem SRCP (125 cm da planta com poda), SRSP (125 cm da planta sem poda), CPR (20 cm da planta com poda), SPR (20 cm da planta sem poda) e T (testemunha)

A amostra de solo coletada no tratamento testemunha, pelo fato de não ter a influência das raízes e ser um solo extremamente salino permaneceu isolado, possivelmente por conta de uma redução drástica da microbiota do solo naquelas condições, tendo em vista que os solos sob a ação da fitoextração por Atriplex nummularia apresentam melhores condições de adaptação e sobrevivência aos microrganismos em solos salinos. Em trabalho realizado com Atriplex nummularia e irrigação destas com rejeitos salinos, Pereira et al. (2004) observaram que houve um aumento da atividade microbiana estimulada pelo plantio da Atriplex.

A diferenciação da atividade biológica no solo em função do posicionamento em relação às plantas de Atriplex nummularia indica que o estabelecimento dessas plantas em solo salino-sódico possibilita a melhoria das condições de desenvolvimento da microbiota associada às plantas e, indiretamente, contribui para a recuperação do mesmo. E o manejo de poda das plantas exerceu influência significativa nesta atividade.

\section{Conclusões}

1. A atividade microbiana no Neossolo Flúvico salinosódico está relacionada com a proximidade do sistema radicular de plantas de Atriplex nummularia;

2. A poda das plantas funcionou como mecanismo de imobilização da microbiota do solo, visando à recuperação de seu estágio inicial de equilíbrio influenciando a atividade microbiana próxima às raízes da planta.

\section{Referências}

AITA, C.; GIACOMINI, S. J. Decomposição e liberação de nitrogênio de resíduos culturais de plantas de cobertura de solo solteiras e consorciadas. Revista Brasileira de Ciência do Solo, v. 27, n. 04 , p. 601-612, 2003.

ALEF, K.; NANNIPIERI, P. (Ed.). Methods in applied soil microbiology and biochemistry. London: Academic Press, 1995. p. 576.

BARRETO, P. A. B. et al. Atividade microbiana, carbono e nitrogênio da biomassa microbiana em plantações de eucalipto, em seqüências de idades. Revista Brasileira de Ciência do Solo, v.32, n. 02, p. 611-619, 2008.

BAYER, C.; MIELNICZUK, J. Dinâmica e função da matéria orgânica. In: SANTOS, G. A.; SILVA, L. S.; CANELLAS, L. P.; CAMARGO, F. A. O. (Eds.). Fundamentos da matéria orgânica do solo: ecossistemas tropicais e subtropicais. Porto Alegre: Metrópole, 2008. Cap. 2, p. 7-16.

BEZERRA, R. G. D., et al. Atividade microbiana em solo cultivado com cana-de-açúcar submetido a doses de fósforo. Revista Verde, v. 03, n. 04, p. 64-69, 2008.

BOT, A.; NACHTERGAELE, F.; YOUNG, A. Land resource potential and constraints at regional and country levels. World Soil Resources Report, 90. Rome: FAO, 2000. 122 p.

BREMNER, J. M.; MULVANEY, R. G. Nitrogen total. In: PAGE, A. L.; MILLER, R. H.; KEENEY, D.R. (Eds.), Methods of Soil Analysis. Madison: American Society of Agronomy, 1982. p. 575- 624.

FERREIRA, D. F. Estatística multivariada. Lavras: Editora UFLA, 2008. 662p.

FLOWERS, T. J. Improving crop salt tolerance. Journal of Experimental botany, v. 55, n. 396, p. 307-319, 2004.

HILL, T.; LEWICKI, P. STATISTICS. Methods and Applications (STATISTICA). Statsoft, Tulsa, OK, 2007.

LEAL, I. G. et al. Fitorremediação de solo salino sódico por Atriplex nummularia e gesso de jazida. Revista Brasileira de Ciência do Solo, v. 32, n. 03, p. 1065-1072, 2008.

MOREIRA, F. M. S.; SIQUEIRA, J. O. Microbiologia e Bioquímica do solo. Lavras: Editora UFLA, 2002. 626 p.

PAULA, J. L.; DUARTE, M. N. Manual de métodos de análise de solo. Rio de Janeiro: Embrapa - CNPS, 1997. 212p.

PEREIRA, S. V. et al. Atividade microbiana em solo do Semi-Árido sob cultivo de Atriplex nummularia. Pesquisa Agropecuária Brasileira, v. 39, n. 08, p. 757-762, 2004.

PORTO, E. R. et al. Rendimento da Atriplex nummularia irrigada com efluentes da criação de tilápia em rejeito da dessalinização de água. Revista Brasileira de Engenharia Agrícola e Ambiental, v. 10, n. 01, p. 97-103, 2006.

SANTOS, P. R. Atributos do solo em função dos diferentes usos adotados em perímetro irrigado do sertão de 
Pernambuco. 2010. 112 f. Tese (Doutorado em Ciência do Solo) - Universidade Federal Rural de Pernambuco, Recife.

SOUZA. E. R. Fitorremediação de Neossolo Flúvico salino sódico de Pernambuco com Atriplex nummularia. 2010. 68 f. Tese (Doutorado em Ciência do Solo) - Universidade Federal Rural de Pernambuco, Recife.

TAYLOR, J. P. et al. Comparison of microbial numbers and enzymatic activities in surface soils and subsoils using various techniques. Soil Biology and Biochemistry, v. 34, n. 03, p. 387-401, 2002.

TEDESCO, M. J. et al. Análise de solo, plantas e outros materiais. Porto Alegre: UFRGS, 1995. 174 p.

TÓTOLA, M. R.; CHAER, G. M. Microrganismos e processos microbiológicos como indicadores da qualidade dos solos. In: CURI, N.; MARQUES, J. J.; GUILHERME, L. R. G.; LIMA, J. M.; LOPES, A. S. S.; ALVAREZ V., V. H., (Eds.) Tópicos em Ciência do Solo. Viçosa, MG, Sociedade Brasileira de Ciência do Solo, 2002. V. 02, p. 195-276.
TU, C.; RISTAINO, J. B.; HU, S. Soil microbial biomass and activity in organic tomato farming systems: effects of organic inputs and straw mulching. Soil Biology and Biochemistry, v. 38, n. 02, p. 247-255, 2006.

VANCE, E. D.; BROOKES, P. C.; JENKINSON, D. S. An extraction method for measuring soil microbial biomass $\mathrm{C}$. Soil biology and Biochemistry, v.19, n. 06, p.703-707, 1987.

VIANA, S. B. A. et al. Índices morfofisiológicos e de produção de alface sob estresse salino. Revista Brasileira de Engenharia Agrícola e Ambiental, v. 08, n. 01, p. 2330,2004

YEOMANS, J. C.; BREMNER, J. M. A rapid and precise method for routine determination of carbon in soil. Communications in Soil Science and Plant Analysis, v. 19, n. 13, p. 1467-1476, 1988.

ZHU, J. K. Plant salt tolerance. Trends in Plant Science, v. 06, n. 02 , p. $66-71,2001$. 